\title{
Mining TCGA Database for Tumor Microenvironment-Related Genes of Prognostic Value in Hepatocellular Carcinoma
}

\author{
Zhenfeng Deng $\mathbb{D},{ }^{1}$ Jilong Wang $\mathbb{D},{ }^{1}$ Banghao Xu $\mathbb{D},{ }^{1}$ Zongrui Jin $\mathbb{D}^{1},{ }^{1}$ Guolin Wu $\mathbb{D}^{1}{ }^{1}$ \\ Jingjing Zeng $\mathbb{D},{ }^{2}$ Minhao Peng $\mathbb{D}^{1},{ }^{1}$ Ya Guo $\mathbb{D}^{1},{ }^{1}$ and Zhang Wen $\mathbb{D}^{1}$ \\ ${ }^{1}$ Department of Hepatobiliary Surgery, The First Affiliated Hospital of Guangxi Medical University, Nanning 530021, China \\ ${ }^{2}$ Department of Pathology, The First Affiliated Hospital of Guangxi Medical University, Nanning 530021, China \\ Correspondence should be addressed to Ya Guo; guoygxmu@163.com and Zhang Wen; wenzgxmu@163.com
}

Received 5 August 2019; Revised 29 September 2019; Accepted 9 October 2019; Published 19 November 2019

Academic Editor: Paul Harrison

Copyright (c) 2019 Zhenfeng Deng et al. This is an open access article distributed under the Creative Commons Attribution License, which permits unrestricted use, distribution, and reproduction in any medium, provided the original work is properly cited.

\begin{abstract}
Hepatocellular carcinoma (HCC) is one of the most common and lethal malignancies. Recent studies reveal that tumor microenvironment (TME) components significantly affect HCC growth and progression, particularly the infiltrating stromal and immune cells. Thus, mining of TME-related biomarkers is crucial to improve the survival of patients with HCC. Public access of The Cancer Genome Atlas (TCGA) database allows convenient performance of gene expression-based analysis of big data, which contributes to the exploration of potential association between genes and prognosis of a variety of malignancies, including HCC. The "Estimation of STromal and Immune cells in MAlignant Tumors using Expression data" algorithm renders the quantification of the stromal and immune components in TME possible by calculating the stromal and immune scores. Differentially expressed genes (DEGs) were screened by dividing the HCC cohort of TCGA database into high- and low-score groups according to stromal and immune scores. Further analyses of functional enrichment and protein-protein interaction networks show that the DEGs are mainly involved in immune response, cell adhesion, and extracellular matrix. Finally, seven DEGs have significant association with HCC poor outcomes. These genes contain FABP3, GALNT5, GPR84, ITGB6, MYEOV, PLEKHS1, and STRA6 and may be candidate biomarkers for HCC prognosis.
\end{abstract}

\section{Introduction}

Hepatocellular carcinoma (HCC) is one of the most common and deadly malignancies worldwide, with approximately 841,000 new cases and 782,000 deaths annually [1]. Currently, the treatment strategies of HCC include surgical resection, transplantation, radiofrequency ablation, transarterial chemoembolization, chemotherapy, and radiotherapy [2]. However, the effectiveness of these therapies is limited in most patients with HCC diagnosed at the middle or advanced stages. A latest study shows that high genetic heterogeneity of HCC may be the main cause of treatment failure [3], and biological and clinical diversities of HCC present great challenges in individualized clinical treatment [4-6]. Genomic heterogeneity in tumor cells has been widely investigated to identify different prognoses and therapeutic responses in subgroups of patients with HCC and to find new molecular targets [7-9]. Moreover, accumulating evidence indicates that nontumor cells in tumor microenvironment (TME) significantly influence the gene expression of tumor cells, which subsequently affects clinical outcomes [9-12]. TME is the microenvironment where the tumor cells are located; other than tumor cells, TME also consists of immune cells, fibroblasts, endothelial cells, extracellular matrix, cytokines, chemokines, and receptors [13]. Stromal and immune cells are two main types of nontumor components in the TME, and the investigation of their interaction has been valuable for developing innovative HCCdirected immunotherapies [14]. However, most previous studies about the HCC TME focused on immune microenvironment and the landscape of stromal cells in the TME lacks in-depth research. Recently, an algorithm that uses 
gene expression signatures to infer the fraction of stromal and immune cells and predict tumor purity in tumor samples has been developed. The algorithm is described as "Estimation of STromal and Immune cells in MAlignant Tumors using Expression data" (ESTIMATE) [15], which can help to understand the landscape of stromal and immune cells in the TME. Some reports have applied the ESTIMATE to colon cancers [16] and glioblastoma [17], revealing the effectiveness of such big-data-based algorithms. However, the effectiveness of stromal and immune scores in HCC has not been elaborated. In this study, we first calculated the stromal and immune scores of HCC cohorts from The Cancer Genome Atlas (TCGA) database by applying the ESTIMATE algorithm and extracted a list of TME-related genes that predict poor outcomes in patients with HCC.

\section{Materials and Methods}

2.1. Data Source. Publicly available dataset of HCC cohort, including Level 3 data of gene expression profile and relevant clinical information, was downloaded from TCGA data portal (https://portal.gdc.cancer.gov/, accessed May 21, 2019). The clinical information includes age, gender, liver fibrosis/cirrhosis status, pathologic stage, histologic grade, values of serum alpha fetoprotein (AFP), Child-Pugh score, microvascular invasion (MVI), radical resection, and survival time. The stromal and immune scores were calculated by applying the ESTIMATE algorithm to the downloaded RNA expression data, and HCC cases were categorized in accordance with the median of immune/stromal scores into high- and low-score groups. All data involved in this study were downloaded from TCGA, and data acquirement and application were performed in accordance with TCGA publication guidelines and data access policies. Thus, additional approval by the local Ethics Committee was not needed.

2.2. Calculation of Stromal and Immune Scores. The stromal and immune scores were calculated by applying the ESTIMATE algorithm to the downloaded RNA expression data, and HCC cases were categorized in accordance with the median of immune/stromal scores into high- and low-score groups. ESTIMATE outputs stromal and immune scores by performing single-sample gene set-enrichment analysis $[15,18]$. For tumor samples of HCC cohort, first, gene expression values were rank-normalized and rank-ordered. Then, the empirical cumulative distribution functions were calculated for genes in the signature and the remaining genes. Finally, a statistic was calculated by an integration of the difference between the empirical cumulative distribution function.

\subsection{Construction of Prognostic Signature Based on Stromal} and Immune Scores. To explore the relevant contribution of stromal and immune scores to HCC survival prediction, they were fitted into a multivariate Cox regression analysis with survival time as the dependent variable. A prognostic risk score model was performed by the linear combination of the stromal and immune scores with the multivariate Cox regression coefficient $(\beta)$ as the weight. The risk score formula was as follows: risk score $=$ stromal score $\times \beta 1+$ immune score $\times \beta 2$ [19]. This prognostic model could divide the HCC cohort into high- and low-risk groups using the median risk score that was based on stromal and immune scores. The time-dependent receiver operating characteristic (ROC) curve was conducted using the "survivalROC" package (version 1.0.3) on the R platform to evaluate the predictive accuracy of this prognostic risk score model [20].

2.4. DEG Screening. Data analysis was performed by using the "limma" package (version 3.40.2) [21] on the R (version 3.6.0). Genes with a mean value $>0$ were included in the screening of DEGs. False discovery rate (FDR) $<0.05$ and $\log 2$ fold change $(\log 2 \mathrm{FC}) \mid \geq 1.5$ were set as the cut-offs to screen for DEGs.

2.5. Functional and Pathway Enrichment Analyses. The DEGs were analyzed by using the "clusterProfiler" R package (version 3.12.0) [22] for Gene Ontology (GO) terms and Kyoto Encyclopedia of Genes and Genomes (KEGG) database pathways. The GO analysis reveals the DEG function in biology process, cell component, and molecular function, and the KEGG analysis shows the pathway enrichment of DEGs. The adjusted $P$ value $<0.05$ was considered statistically significant.

2.6. Construction of Protein-Protein Interaction (PPI) Network. The PPI network of DEGs was retrieved through the Search Tool for the Retrieval of Interacting Genes (STRING, https://string-db.org/) [23] and reconstructed via Cytoscape software (version 3.7.1) [24], which is an opensource software platform for visualizing complex networks and integrating these with any type of attribute data. The Molecular Complex Detection plugin of Cytoscape was then used to find the most significant module based on topology to locate densely connected regions. The settings of selection were as follows: degree cut-off $=2$, node score cut-off $=0.2$, $\mathrm{k}$ - core $=2$, and maximum depth $=100$.

2.7. Statistical Analysis. Kaplan-Meier survival analysis by log-rank test was used to identify the TME-related DEGs regarding HCC poor prognosis. Univariate analyses between clinical characteristics and stromal/immune scores were compared using the log-rank test. A value of $P<0.05$ was considered statistically significant. Venn diagrams, heat maps, and survival curves were plotted by $\mathrm{R}$ platform. Statistical analysis was performed using SPSS 22.0 (Chicago, IL, USA).

\section{Results}

3.1. Study Population and Their Stromal and Immune Scores. A total of 374 cases were available from the TCGA database for further analysis. Their RNA expression data of tumor tissues were used to calculate the stromal and immune 
scores. Based on the ESTIMATE algorithm, the range of stromal score was from $-1,625.38$ to $1,171.12$, and the range of immune score was from -866.31 to $3,146.06$. The relevant clinical data were also downloaded to investigate correlation with stromal and immune scores (Table 1). Univariate analysis identified the following clinical features as significantly associated with stromal scores: histologic grade (log-rank $P=0.014)$, serum AFP (log-rank $P=0.013)$, and MVI (logrank $P=0.018$ ), and liver fibrosis/cirrhosis status was also significantly associated with immune scores (log-rank $P=0.013)$. To explore the potential correlation of overall survival (OS) with stromal and immune scores, 374 HCC cases were divided into high- and low-score groups in accordance with the stromal/immune scores. Survival analysis shows that the median survival time (MST) of cases in the low-score group of stromal scores is longer than that in the high-score group (MST: 453 vs. 631 days; $\log$-rank $P=0.256$ ). Cases with lower immune scores also showed longer MST compared with cases with higher immune scores (MST: 500 vs. 602 days; log-rank $P=0.377$ ), but both were not statistically significant at the $P<0.05$ level and relevant figures are not shown. A multivariate Cox regression analysis was applied to further assess the relative contribution of the stromal and immune scores in survival prediction. The risk score formula was as follows: risk score $=$ stromal score $\times(-0.1074)+$ immune score $\times(-0.4074)$. Survival analysis shows that patients with a high-risk score have a shorter MST than those with a low-risk score in 1-year OS (MST: 299 vs. 311 days; $\log$-rank $P=0.332$; Figure 1(a)), 3-year OS (MST: 545 vs. 633 days; $\log$-rank $P=0.037$; Figure 1(b)), and 5-year OS (MST: 666 vs. 763 days; log-rank $P=0.180$; Figure 1(c)). The area under the curve (AUC) of ROC curve was $0.577,0.625$, and 0.625 for 1-, 3-, and 5-year survival based on the time-dependent ROC analysis (Figures 1(d)-1(f)).

3.2. DEG Screening. Unique gene expression profiles of 374 cases were shown in heat maps by categorizing the HCC cohort into high- and low-scores groups of stromal/immune cells (Figures 2(a) and 2(b)). For comparing high and low groups based on the stromal scores, 584 upregulated genes and 32 downregulated genes were identified. For the immune score groups, 583 upregulated genes and 31 downregulated genes were identified. In addition, Venn diagrams showed that 281 identical genes were upregulated and 8 identical genes were downregulated between the stromal and immune score groups (Figures 2(c) and 2(d)). Therefore, a total of 289 genes were screened as DEGs after taking the intersection by drawing Venn diagrams, which meets the criteria of FDR $<0.05$ and $|\log 2 \mathrm{FC}| \geq 1.5$.

3.3. Functional Assessment. Functional enrichment analysis was performed for DEGs by applying the clusterProfiler R package, which shows that these DEGs were highly correlated with immune response. GO term enrichment analysis (adjusted $P$ value $<0.05$; Figure 3(a)) indicated that DEGs were significantly enriched in the biological processes of immune cell differentiation and activation, cell component of extracellular matrix and membrane, molecular function of surface receptor activity, and protein binding. Moreover, the
KEGG analysis (adjusted $P$ value $<0.05$; Figure 3(b)) suggested that most of DEG-related pathways were significantly linked to immune response.

3.4. Module Analysisfrom the PPI Network. The PPI network of DEGs was acquired by applying the online STRING tool. This network consists of nine modules, which include 74 nodes and 255 edges. The top three significant modules were selected for further analysis (Figure 4). We named these modules as Modules 1, 2, and 3, respectively. In Module 1, 15 nodes with 105 edges were formed in the network, including ADRA2A, CCL19, CCL21, CCR4, CCR5, CCR7, CXCL9, CXCR1, CXCR2, CXCR6, FPR1, FPR3, GPR183, P2RY12, and $P 2 R Y 13$ (Figures 4(a) and 4(b)). Module 2 contained 54 edges involving 13 nodes: BTLA, CD163, CD2, CD22, CD40LG, CD5, CD69, CD80, CR2, ITGA4, PTPRC, SPN, and TNFRSF8 (Figures 4(c) and 4(d)). Module 3 included 42 edges involving 14 nodes: $C D 1 B, C D 3 E, C D 48, C D 52$, HAVCR2, IKZF1, IL10, IL2RA, IL7R, ITK, LCK, SELL, TLR7, and TLR8 (Figures 4(e) and 4(f)). The KEGG enrichment analysis showed that the genes in Modules 1 to 3 are mainly correlated with chemokine signaling pathway, cell adhesion molecules, and hematopoietic cell lineage.

3.5. Survival Analysis. To explore the underlying prognostic value of individual DEGs, the survival analysis was performed between 289 DEGs and the OS in patients with HCC from TCGA database (Table S1). Among the 289 DEGs, a total of 12 DEGs were shown to significantly associate with poor OS (log-rank $P<0.05$ ), which contain CD80, FABP3, GALNT5, GPR84, IL11, ITGB6, MMP7, MMP12, MYEOV, PLEKHS1, PTGIS, and STRA6 (Figure 5). All the 12 genes were upregulated DEGs.

\section{Discussion}

The data mining of TCGA database has been widely applied to cancer prognosis prediction, and recent studies reveal that TME plays a crucial role in HCC growth and progression $[9,25]$. Therefore, we intend to identify TME-related genes that significantly affect HCC prognosis from TCGA database in this study. Particularly, these genes associate with stromal and immune components in the TME.

First, we acquired the stromal and immune scores to determine whether they were associated with the clinical characteristics and OS of HCC patients. The results show that they were indeed related to the indicators of clinical progress and prognosis, such as liver fibrosis/cirrhosis status, MVI, histological grade, and serum AFP. The multivariate Cox regression model based on stromal and immune scores showed that the risk score was significantly associated with 3 -year OS, and time-dependent ROC analysis demonstrated that this prognostic risk score model performed well in 3year OS prediction. Next, 289 DEGs were screened by comparing the high- and low-score groups of stromal and immune cells. Subsequent GO term analysis found that most of them were involved in TME, and the KEGG pathways analysis also shows that most of the DEGs were 
TABle 1: Distribution of HCC patients' characteristics and their clinical correlation with stromal and immune scores.

\begin{tabular}{|c|c|c|c|c|c|}
\hline \multirow{2}{*}{ Variables } & \multirow{2}{*}{ Count (total $n=374$ ) } & \multicolumn{2}{|c|}{ Stromal scores } & \multicolumn{2}{|c|}{ Immune scores } \\
\hline & & Median & $P$ value & Median & $P$ value \\
\hline Age (years) & & & 0.488 & & 0.868 \\
\hline$\leq 60$ & $177(47.3 \%)$ & -661.51 & & 416.99 & \\
\hline$>60$ & $193(51.6 \%)$ & -707.93 & & 437.03 & \\
\hline NA & $4(1.1 \%)$ & - & & - & \\
\hline Gender & & & 0.940 & & 0.326 \\
\hline Female & $121(32.4 \%)$ & -667.77 & & 386.47 & \\
\hline Male & $250(66.8 \%)$ & -690.11 & & 441.65 & \\
\hline NA & $3(0.8 \%)$ & - & & - & \\
\hline Liver fibrosis/cirrhosis & & & 0.565 & & $0.013^{\mathrm{a}}$ \\
\hline No & $74(19.8 \%)$ & -713.70 & & 243.15 & \\
\hline Yes & $138(36.9 \%)$ & -657.35 & & 522.19 & \\
\hline NA & $162(43.3 \%)$ & - & & - & \\
\hline Pathologic stage & & & 0.180 & & 0.084 \\
\hline Stage I & $171(45.7 \%)$ & -656.40 & & 454.29 & \\
\hline Stage II & $86(23.0 \%)$ & -748.34 & & 503.16 & \\
\hline Stages III and IV & $90(24.1 \%)$ & -752.87 & & 306.66 & \\
\hline NA & $27(7.2 \%)$ & - & & - & \\
\hline Histologic grade & & & $0.014^{\mathrm{a}}$ & & 0.938 \\
\hline G1 & $55(14.7 \%)$ & -385.71 & & 307.42 & \\
\hline G2 & $177(47.3 \%)$ & -658.31 & & 459.21 & \\
\hline G3 & $122(32.6 \%)$ & -771.93 & & 424.43 & \\
\hline G4 & $12(3.2 \%)$ & -1041.72 & & 540.85 & \\
\hline NA & $8(2.2 \%)$ & - & & - & \\
\hline Serum AFP & & & $0.013^{\mathrm{a}}$ & & 0.533 \\
\hline$\leq 400 \mathrm{ng} / \mathrm{mL}$ & $213(56.9 \%)$ & -647.06 & & 458.80 & \\
\hline$>400 \mathrm{ng} / \mathrm{mL}$ & $65(17.4 \%)$ & -824.84 & & 447.73 & \\
\hline NA & $96(25.7 \%)$ & - & & - & \\
\hline Child-Pugh score & & & 0.350 & & 0.207 \\
\hline A & $217(58.0 \%)$ & -697.06 & & 416.70 & \\
\hline $\mathrm{B}$ and $\mathrm{C}$ & $22(5.9 \%)$ & -790.82 & & 158.16 & \\
\hline NA & $135(36.1 \%)$ & - & & - & \\
\hline$M V I$ & & & $0.018^{\mathrm{a}}$ & & 0.644 \\
\hline No & $206(55.1 \%)$ & -590.14 & & 424.57 & \\
\hline Yes & $109(29.1 \%)$ & -755.81 & & 416.70 & \\
\hline NA & $59(15.8 \%)$ & - & & - & \\
\hline Radical resection & & & 0.478 & & 0.552 \\
\hline $\mathrm{R} 0$ & $324(86.6 \%)$ & -703.78 & & 412.96 & \\
\hline $\mathrm{R} 1 \& \mathrm{R} 2$ & $18(4.8 \%)$ & -527.29 & & 467.77 & \\
\hline NA & $32(8.6 \%)$ & - & & - & \\
\hline
\end{tabular}

a The value of $P<0.05$ indicates statistical significance; NA: not available.

significantly associated with immune response, which is consistent with previous reports stating that the functions of stromal and immune components are interrelated in constituting TME in HCC $[12,14,26]$. Then, we constructed the PPI network to better understand the interactions of DEGs, and the top three modules show that they were all significantly related to the pathway of immunologic and inflammatory response. CCR7, PTPRC (CD45), and IL10 were the most connected nodes in these modules, in which CCR7 was a crucial molecule in the mechanism of HCC's progression and metastasis [27-29], PTPRC involved in the regulation of cytokine-induced signaling in malignancies [30-32], and IL10 has been reported to increase the susceptible risk of HCC [33], decrease immunologic activity [34], and promote immune tolerance in the tumor milieu [35, 36]. Finally, survival analysis was performed to explore the potential prognostic value of 289 DEGs, and we identified 12 TME-related genes that showed significant correlation between gene expression and poor outcomes in HCC cases. Of the 12 genes, five genes (CD80, IL11, MMP7, MMP12, and PTGIS) have been reported to be associated with HCC's progression or significant in HCC survival prediction [37-42], indicating that our big data analysis based on ESTIMATE algorithm has prognostic values in the HCC cohort of TCGA database. The other seven genes have never been reported to correlate with HCC development and prognosis before and can be perceived as potential biomarkers for HCC.

Among the seven potential biomarkers FABP3, GALNT5, GPR84, ITGB6, MYEOV, PLEKHS1, and STRA6, 


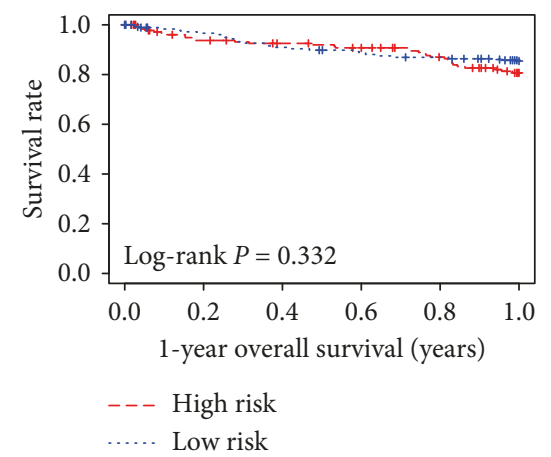

(a)

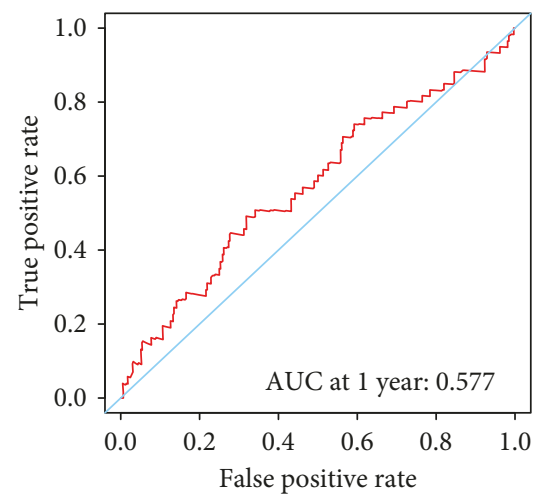

(d)

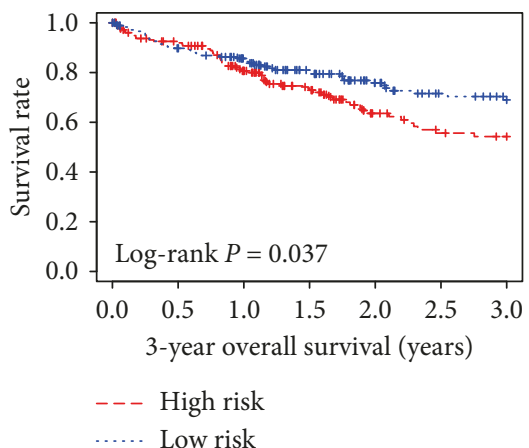

(b)

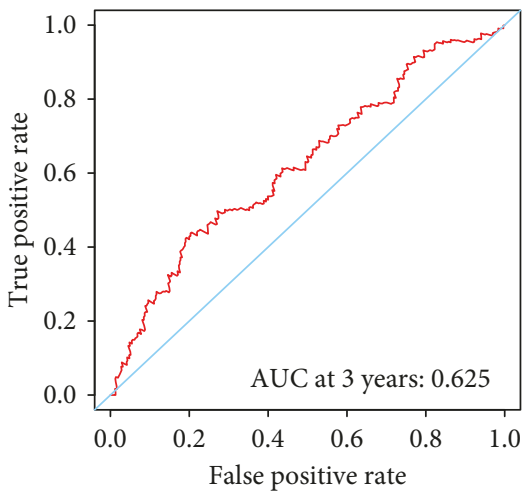

(e)

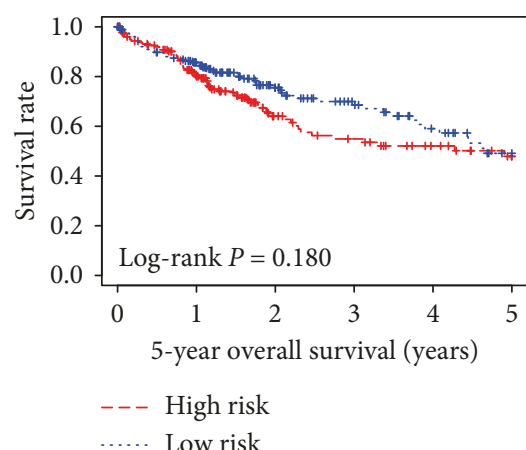

(c)

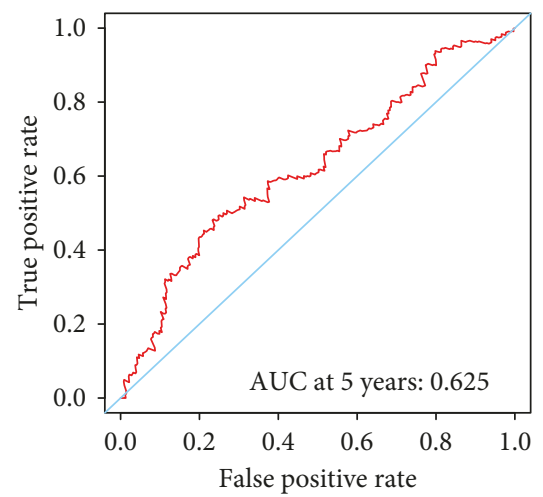

(f)

Figure 1: The Kaplan-Meier and ROC curves of prognostic risk score model based on stromal and immune scores in HCC. Kaplan-Meier curves of high- and low-risk groups for 1-year OS (a), 3-year OS (b), and 5-year OS (c); ROC curves of risk score model for 1-year OS (d), 3year OS (e), and 5-year OS (f).

we are particularly interested in GPR84 and STRA6 because they link to liver fibrosis that is a major risk factor in HCC and an independent risk factor of recurrence after hepatectomy $[43,44]$. GPR84, a protein-coding gene of the metabolic G protein-coupled receptor family, plays a potential role in the lipid metabolism and regulation of inflammation. The latest study demonstrates that GPR84 is involved in fibrotic pathway and Gpr84 knockout model in mice can reduce the degree of fibrosis [45]. Moreover, targeted GPR84 treatment has been shown to be effective in liver fibrosis [46]. Therefore, GPR84 enables the promotion of liver fibrosis and is deleterious in chronic liver disease, which may be the cause of pathogenesis and progression in HCC. Likewise, STRA6, as a coding gene of membrane protein involved in the metabolism of retinol, is also reported to be involved in relevant signaling of fibrosis [47] and has been shown to inhibit the effectiveness of antifibrotic treatment [48]. Interestingly, fibrosis is characterized by the excessive accumulation of extracellular matrix in damaged or inflamed tissues [49], which indicates from another aspect that GPR84 and STRA6 are related to TME component. The other five candidate genes are FABP3, GALNT5, ITGB6, MYEOV, and PLEKHS1. FABP3 belongs to the intracellular fatty acid-binding protein family, which is thought to participate in the uptake, intracellular metabolism, and transport of long-chain fatty acids, and may be responsible for the modulation of cell growth and proliferation [50]. Recent study has suggested that FABP3 is upregulated in hepatic steatosis in zebrafish model, and hepatic steatosis can be ameliorated by suppressing FABP3 expression in the liver [51]. Hepatic steatosis, like the nonalcoholic fatty liver disease, is also a major risk factor for HCC. Thus, further investigation is necessary to identify the potential biological relevance between FABP3 and HCC. GALNT5 encodes a membrane-bound transferase in the Golgi and is reported to facilitate the proliferation and migration of colorectal and gastric cancer cells [52, 53]. ITGB6 encodes a protein that is a member of the integrin superfamily. Members of this family are adhesion receptors that function in signaling from the extracellular matrix to the cell. A study demonstrates that ITGB6 is expressed in malignant colonic epithelia and is associated with the progression, metastasis, and chemotherapeutic resistance of colon cancer [54]. MYEOV gene is localized at chromosome $11 \mathrm{q} 13$ that is a frequent site for chromosomal rearrangements in various carcinomas and B-cell neoplasms [55]. Previous reports suggest that the expression of MYEOV is enhanced in non-small-cell lung cancer and colorectal cancer and promotes cancer cell proliferation and invasion $[56,57]$. For PLEKHS1, the proteins encoded by PLEKHS1 participate in intracellular signaling. Meanwhile, PLEKHS1 is able to cause noncoding mutations by regulating recurrent mutations of upstream and promoter elements, which can lead to tumorigenesis [58]. Currently, although the 


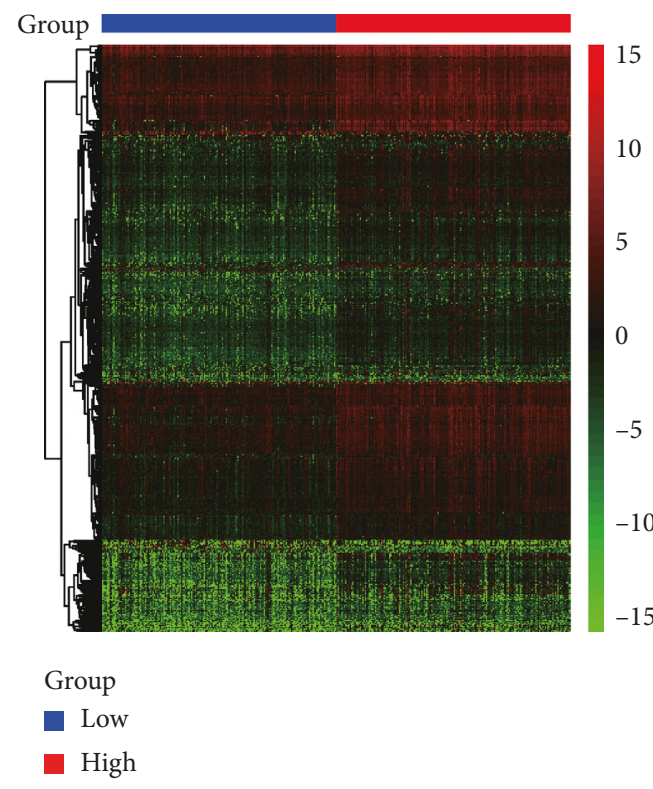

(a)

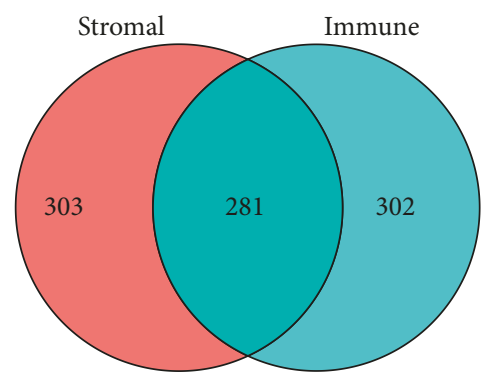

(c)

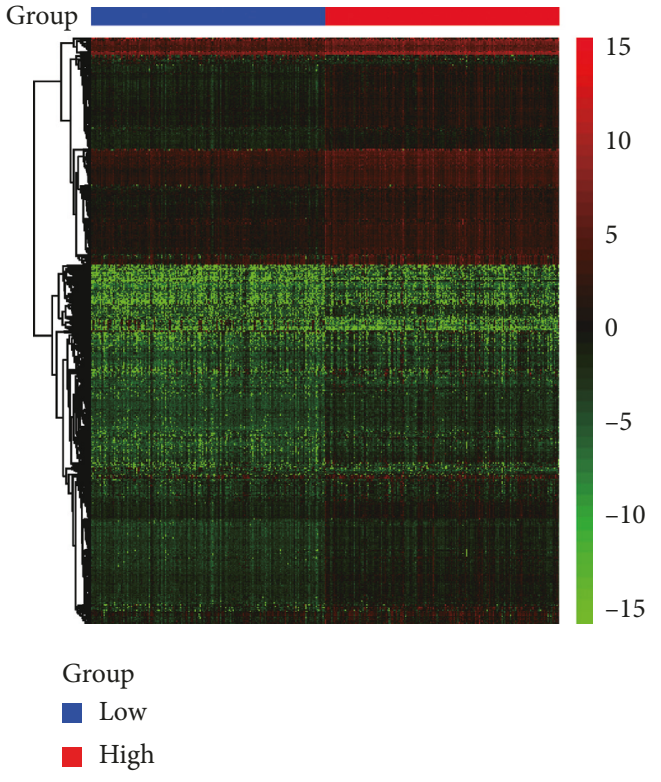

(b)

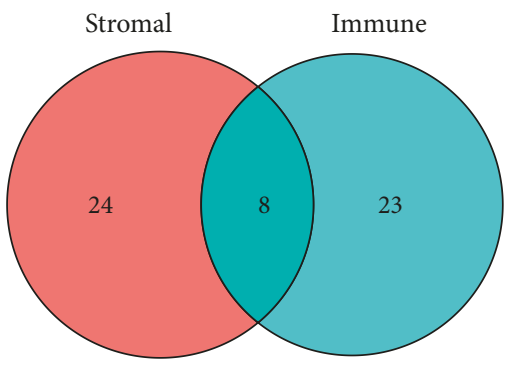

(d)

Figure 2: Comparison of gene expression profile with stromal and immune scores in HCC. Heat map of DEGs between high and low groups in (a) stromal scores and (b) immune scores. In drawn heat maps, genes with higher expression are shown in red, while lower expressions are shown in green, and the same expression levels are in black. Venn diagrams show the number of (c) co-upregulated or (d) co-downregulated genes between stromal and immune groups.

functional verification experiments of the seven genes in HCC have not been reported, they are more or less associated with the occurrence and development of malignancies and still need further investigation.

The studies reported in the past decade have been able to delineate the landscape of genomic alterations and gene signatures occurring in HCC growth and progression [59]. This delineation has certainly changed our perception of the disease. Meanwhile, great development has been made on the correlation of prognostic prediction with the gene expression in HCC. Many of these studies were performed through the construction of animal models, experiments of cell in vitro, and small-scale cohorts of clinical tumor samples. However, the complex interplay of HCC and the microenvironment where it is located demands a highly comprehensive analysis of large-scale cohorts. Fortunately, due to the significant progress of whole-genome sequencing technology, some high-throughput tumor databases, such as TCGA, have been developed and are publicly available for open academic communication. These platforms can provide resources for big data analysis with large-scale cohorts of HCC or other malignancies.

Compared with previous reports that focused on how the activation of tumor intrinsic gene exerts an influence on the TME, our study attaches high importance to gene signatures in TME, which in turn act on HCC's development, hence affecting patients' prognosis. Our study may provide additional data and new ideas to analyze the complex interactions between HCC and the TME where it is located. However, some limitations exist in this study which still needs to be elaborated. First the clinical information from the TCGA database is incomplete, such that detailed data about the treatment after surgery are unavailable. As a result, we were not able to perform a comprehensive survival analysis that considered other potential prognostic factors in HCC. Second, HCC cases in this study are exclusively obtained from a single cohort, which may have caused biases to our results. Third, our findings still need further validation, which may be performed through confirmatory experiments with realtime PCR and Western blot for screened genes. 


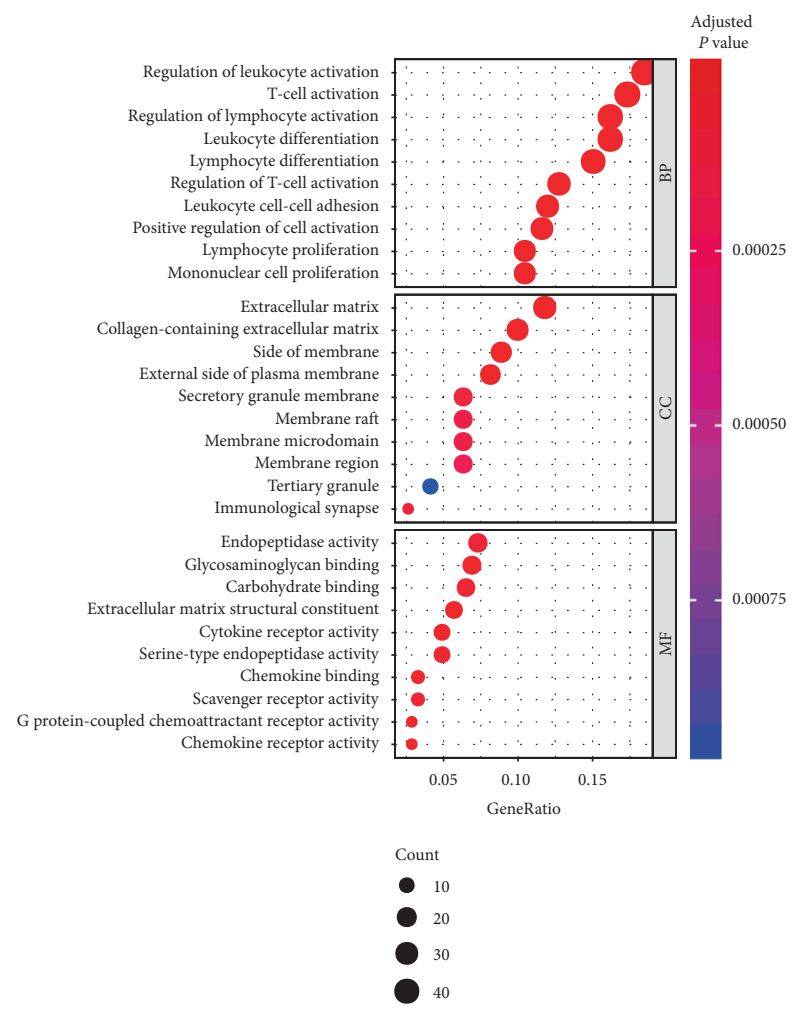

(a)

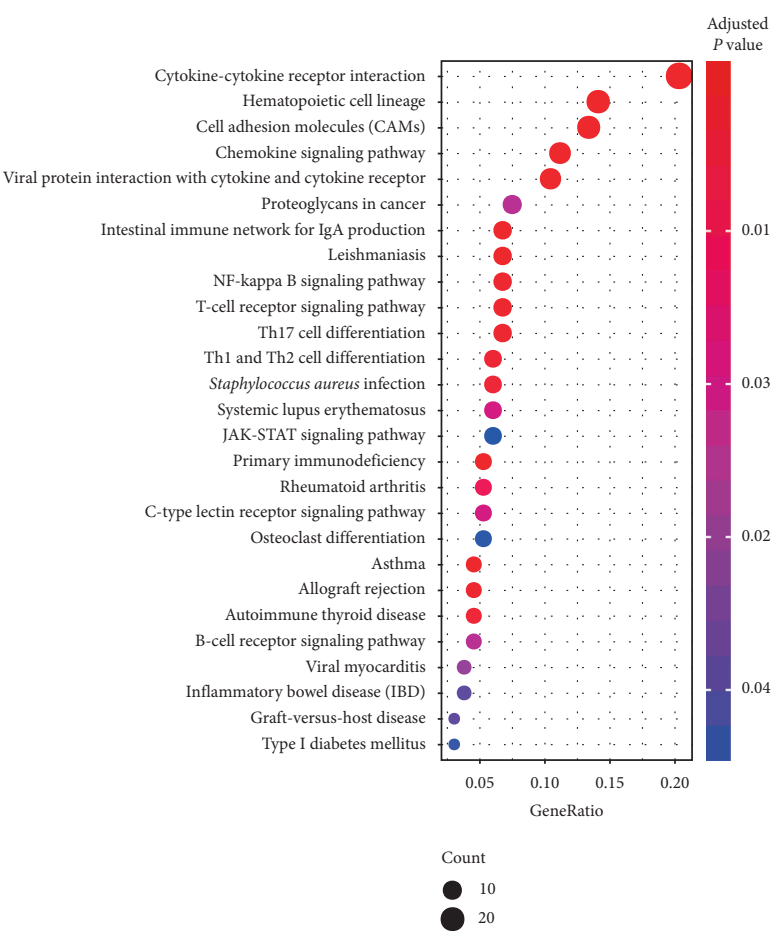

(b)

FIGURE 3: Functional enrichment analysis results of DEGs. (a) GO term enrichment results. (b) KEGG enrichment results.

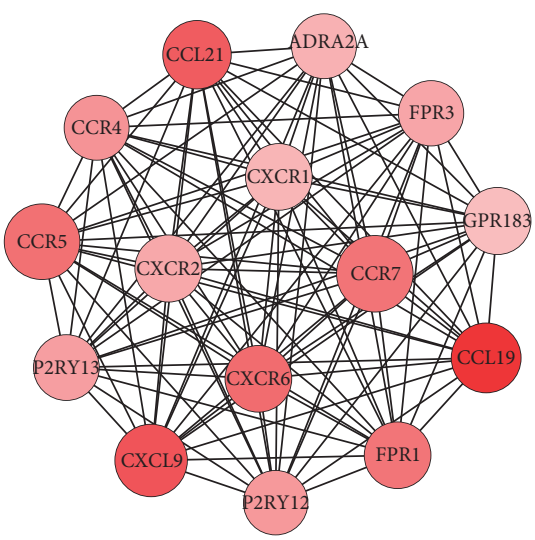

$\log 2 \mathrm{FC}$

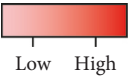

Count

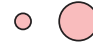

Low High

(a)

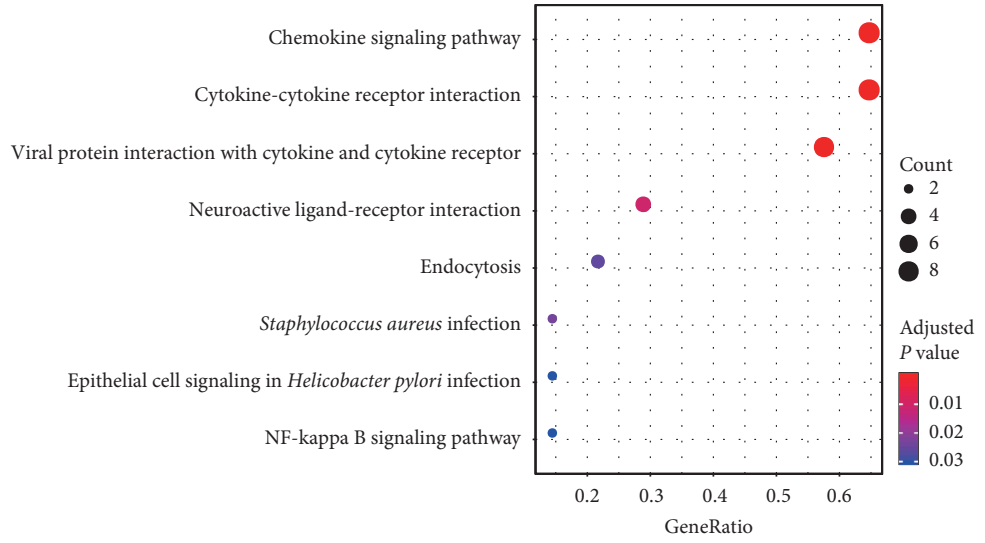

(b)

FIgURE 4: Continued. 

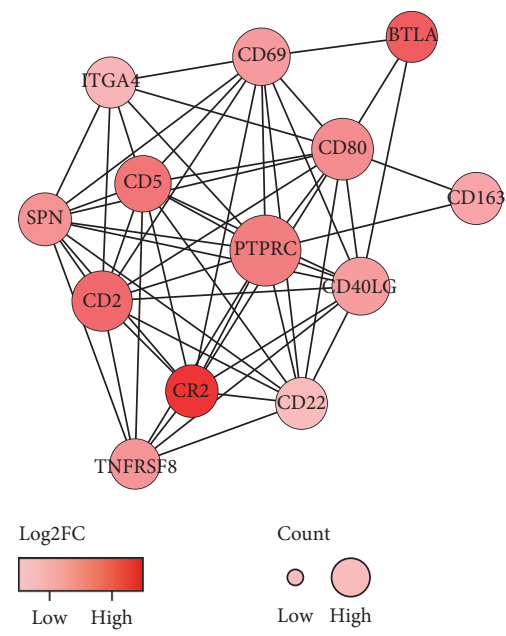

(c)

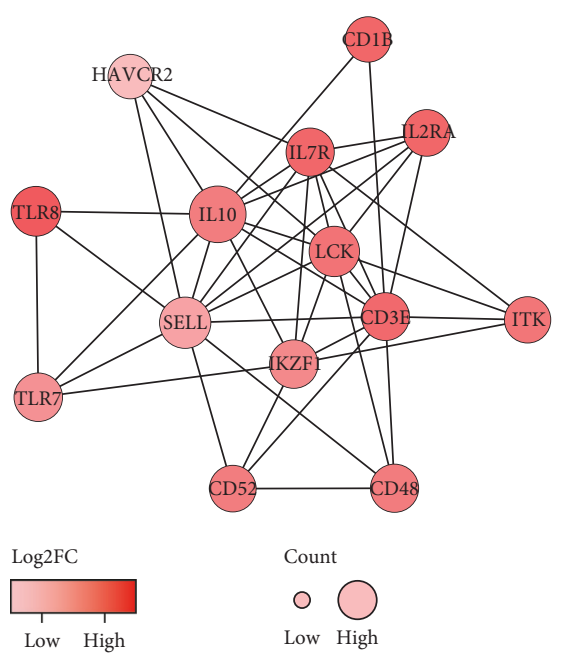

(e)

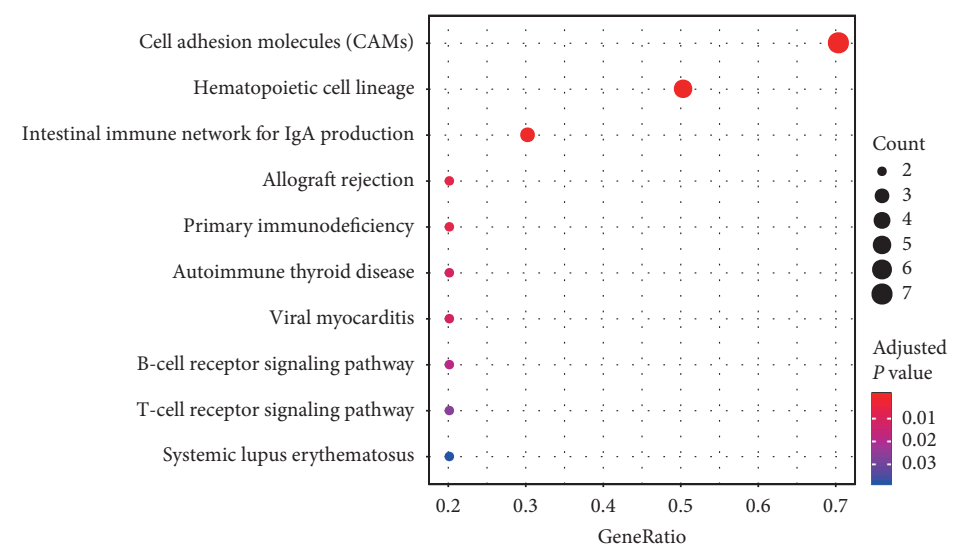

(d)

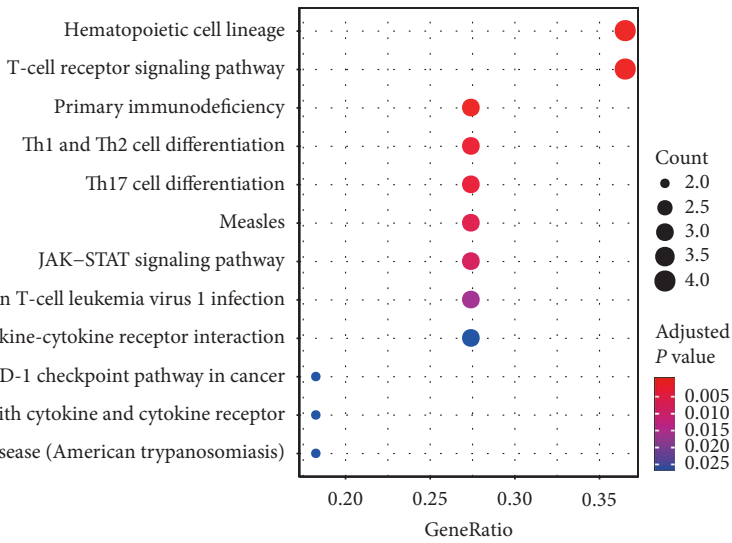

(f)

FIgURE 4: The top three significant modules from PPI network and their pathway enrichment analysis. (a) Module 1. (b) KEGG enrichment results of Module 1. (c) Module 2. (d) KEGG enrichment results of Module 2. (e) Module 3. (f) KEGG enrichment results of Module 3. In the modules, the color of a node in the PPI network reflects the log2FC value of gene expression, and the size of node indicates the count of edges with other genes.

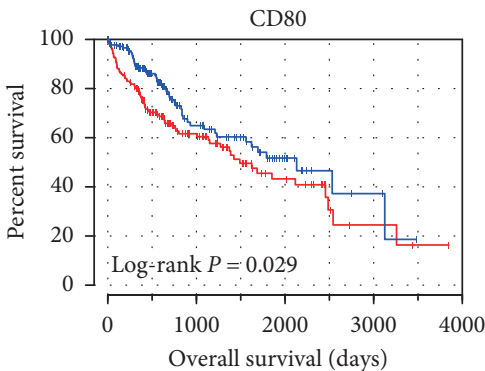

$$
\begin{array}{ll}
\text { Expression } & \text { Expression } \\
- \text { High } & - \text { High }
\end{array}
$$

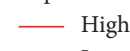

(a)

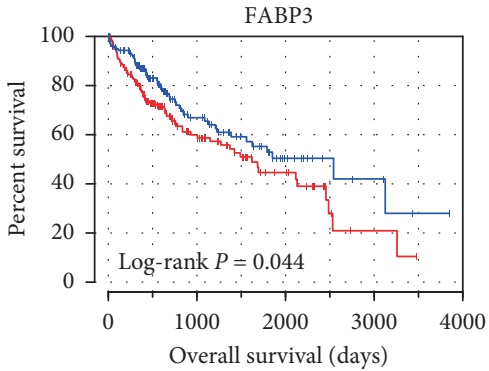

(b)

Figure 5: Continued. 

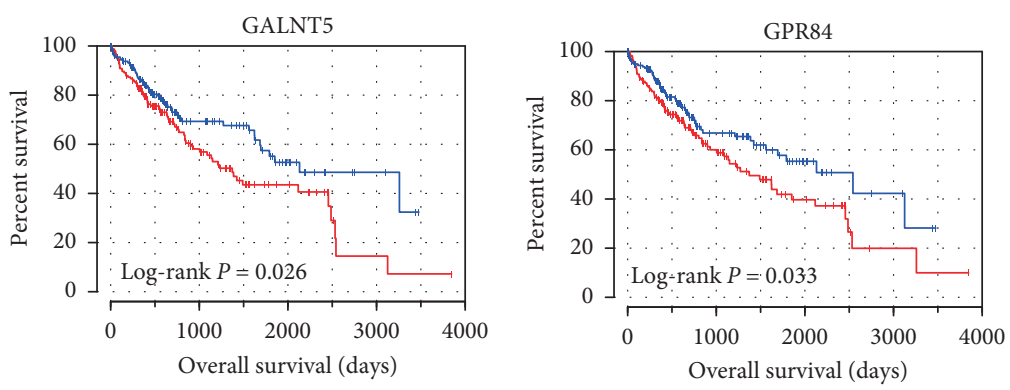

Expression

Expression

High
Low

- High

(c)

(d)

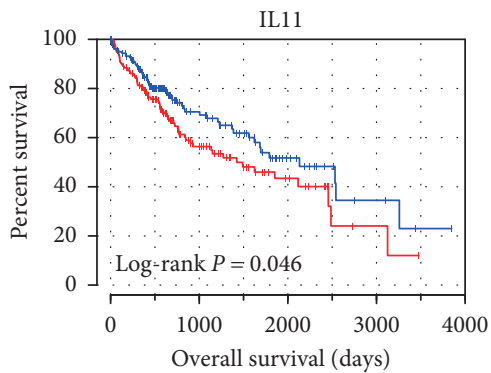

ITGB6

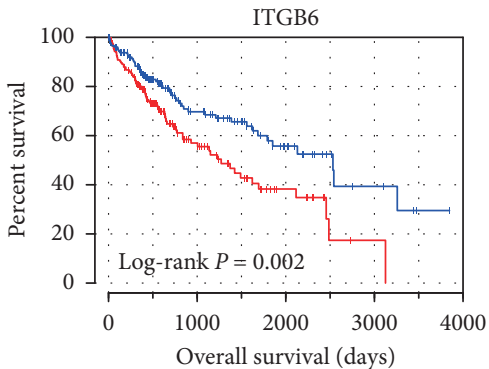

Expression

— High

Expression

- High

(e)

(f)

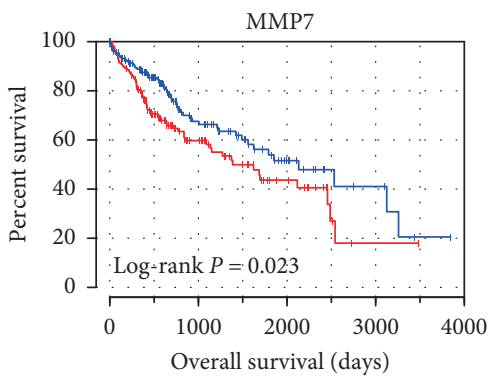

MMP12

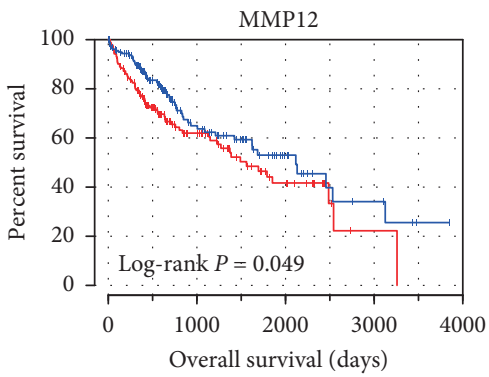

Expression

Expression

— High

- High

_ Low

(g)

(h)
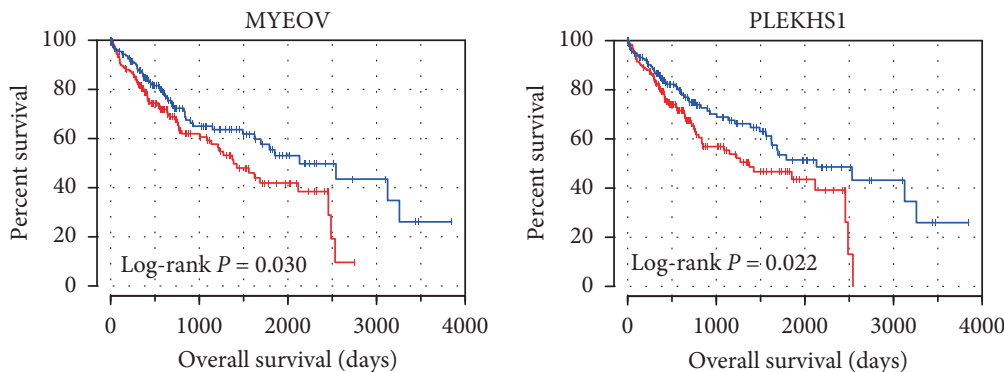

Expression

Expression

- High

High

_ Low

(i)

FIgURE 5: Continued. 


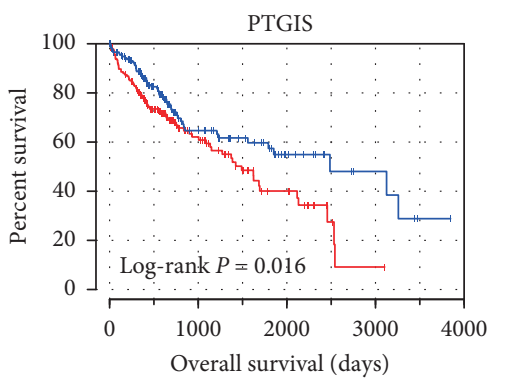

Expression High
- Low

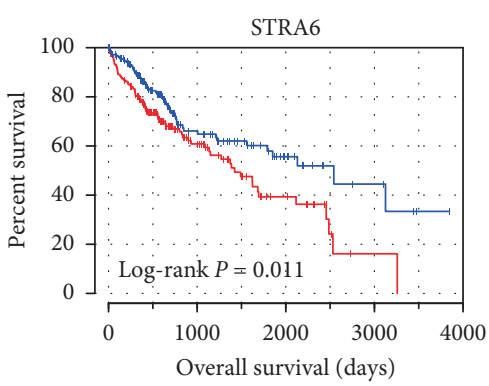

Expression

- High

$(\mathrm{k})$

(1)

FIgURE 5: Survival analysis between DEGs and poor OS in HCC. Kaplan-Meier curves were drawn for screening DEGs with prognostic value from the comparison between high (red line) and low (blue line) gene expression groups.

\section{Conclusions}

In conclusion, we calculated the stromal and immune scores based on the ESTIMATE algorithm to identify 12 TMErelated genes with poor prognosis from the HCC cohort of TCGA database. Seven of the 12 genes, namely, FABP3, GALNT5, GPR84, ITGB6, MYEOV, PLEKHS1, and STRA6, can be perceived as candidate genes for the prognostic prediction of HCC, which have not been previously reported for their prognostic value in HCC patients. Further study on these genes can contribute to an in-depth and comprehensive understanding of the potential correlation between TME and HCC prognosis. In addition, we anticipate that our strategy of mining TME-related genes can be widely applied to big data analysis and discover more biomarkers with prognosis value for HCC or other malignancies.

\section{Data Availability}

The datasets analyzed during the current study are available from the corresponding author on reasonable request.

\section{Conflicts of Interest}

The authors declare that there are no conflicts of interest regarding the publication of this article.

\section{Authors' Contributions}

Zhenfeng Deng and Jilong Wang contributed equally to this work. Zhenfeng Deng and Jilong Wang designed the study protocol. Jilong Wang, Banghao Xu, Zongrui Jin, Guolin Wu, Jingjing Zeng, and Minhao Peng conducted the study and analyzed the data. Zhenfeng Deng wrote the manuscript. Zhang Wen and Ya Guo revised the manuscript. Zhang Wen, Ya Guo, and Jilong Wang strived for financial support.

\section{Acknowledgments}

This study was financially supported by the National Natural Science Foundation of China (Grant no. 81560387), the
Guangxi Natural Science Foundation of China (Grant no. 2018GXNSFBA050030), and the "Medical Excellence Award" funded by the Creative Research Development Grant from the First Affiliated Hospital of Guangxi Medical University (Grant no. 180327).

\section{Supplementary Materials}

Table S1: the survival analysis results of the 289 DEGs. (Supplementary Materials)

\section{References}

[1] F. Bray, J. Ferlay, I. Soerjomataram, R. L. Siegel, L. A. Torre, and A. Jemal, "Global cancer statistics 2018: GLOBOCAN estimates of incidence and mortality worldwide for 36 cancers in 185 countries," CA: A Cancer Journal for Clinicians, vol. 68, no. 6, pp. 394-424, 2018.

[2] J. K. Heimbach, L. M. Kulik, R. S. Finn et al., "AASLD guidelines for the treatment of hepatocellular carcinoma," Hepatology, vol. 67, no. 1, pp. 358-380, 2018.

[3] Q. Gao, Z.-C. Wang, M. Duan et al., "Cell culture system for analysis of genetic heterogeneity within hepatocellular carcinomas and response to pharmacologic agents," Gastroenterology, vol. 152, no. 1, pp. 232-242, 2017.

[4] K.-S. Jeng, C.-F. Chang, W.-J. Jeng, I.-S. Sheen, and C.-J. Jeng, "Heterogeneity of hepatocellular carcinoma contributes to cancer progression," Critical Reviews in Oncology/Hematology, vol. 94, no. 3, pp. 337-347, 2015.

[5] V. Thorsson, D. L. Gibbs, S. D. Brown et al., "The immune landscape of cancer," Immunity, vol. 48, no. 4, pp. 812-830, 2018.

[6] A. Forner, M. Reig, and J. Bruix, "Hepatocellular carcinoma," The Lancet, vol. 391, no. 10127, pp. 1301-1314, 2018.

[7] D.-C. Lin, A. Mayakonda, H. Q. Dinh et al., "Genomic and epigenomic heterogeneity of hepatocellular carcinoma," Cancer Research, vol. 77, no. 9, pp. 2255-2265, 2017.

[8] A. Huang, X. Zhao, X.-R. Yang et al., "Circumventing intratumoral heterogeneity to identify potential therapeutic targets in hepatocellular carcinoma," Journal of Hepatology, vol. 67, no. 2, pp. 293-301, 2017.

[9] J. D. Yang, I. Nakamura, and L. R. Roberts, "The tumor microenvironment in hepatocellular carcinoma: current 
status and therapeutic targets," Seminars in Cancer Biology, vol. 21, no. 1, pp. 35-43, 2011.

[10] C. J. Lim, Y. H. Lee, L. Pan et al., "Multidimensional analyses reveal distinct immune microenvironment in hepatitis B virus-related hepatocellular carcinoma," Gut, vol. 68, no. 5, pp. 916-927, 2019.

[11] Y. Kurebayashi, H. Ojima, H. Tsujikawa et al., "Landscape of immune microenvironment in hepatocellular carcinoma and its additional impact on histological and molecular classification," Hepatology, vol. 68, no. 3, pp. 1025-1041, 2018.

[12] S. J. Turley, V. Cremasco, and J. L. Astarita, "Immunological hallmarks of stromal cells in the tumour microenvironment," Nature Reviews Immunology, vol. 15, no. 11, pp. 669-682, 2015.

[13] H. Bolouri, "Network dynamics in the tumor microenvironment," Seminars in Cancer Biology, vol. 30, pp. 52-59, 2015.

[14] M. Sprinzl and P. Galle, "Immune control in hepatocellular carcinoma development and progression: role of stromal cells," Seminars in Liver Disease, vol. 34, no. 4, pp. 376-388, 2014.

[15] K. Yoshihara, M. Shahmoradgoli, E. Martínez et al., "Inferring tumour purity and stromal and immune cell admixture from expression data," Nature Communications, vol. 4, p. 2612, 2013.

[16] M. H. Alonso, S. Aussó, A. Lopez-Doriga et al., "Comprehensive analysis of copy number aberrations in microsatellite stable colon cancer in view of stromal component," British Journal of Cancer, vol. 117, no. 3, pp. 421-431, 2017.

[17] D. Jia, S. Li, D. Li, H. Xue, D. Yang, and Y. Liu, "Mining TCGA database for genes of prognostic value in glioblastoma microenvironment," Aging, vol. 10, no. 4, pp. 592-605, 2018.

[18] R. G. W. Verhaak, K. A. Hoadley, E. Purdom et al., "Integrated genomic analysis identifies clinically relevant subtypes of glioblastoma characterized by abnormalities in PDGFRA, IDH1, EGFR, and NF1," Cancer Cell, vol. 17, no. 1, pp. 98-110, 2010.

[19] X. Liao, X. Wang, K. Huang et al., "Genome-scale analysis to identify prognostic microRNA biomarkers in patients with early stage pancreatic ductal adenocarcinoma after pancreaticoduodenectomy," Cancer Management and Research, vol. 10, pp. 2537-2551, 2018.

[20] C. Combescure, T. V. Perneger, D. C. Weber, J. P. Daurès, and Y. Foucher, "Prognostic ROC curves: a method for representing the overall discriminative capacity of binary markers with right-censored time-to-event endpoints," Epidemiology (Cambridge, Mass), vol. 25, no. 1, pp. 103-109, 2014.

[21] M. E. Ritchie, B. Phipson, D. Wu et al., "Limma powers differential expression analyses for RNA-sequencing and microarray studies," Nucleic Acids Research, vol. 43, no. 7, p. e47, 2015.

[22] G. Yu, L.-G. Wang, Y. Han, and Q.-Y. He, "clusterProfiler: an $\mathrm{R}$ package for comparing biological themes among gene clusters," OMICS: A Journal of Integrative Biology, vol. 16, no. 5, pp. 284-287, 2012.

[23] D. Szklarczyk, A. L. Gable, D. Lyon et al., "STRING v11: protein-protein association networks with increased coverage, supporting functional discovery in genome-wide experimental datasets," Nucleic Acids Research, vol. 47, no. 1, pp. D607-D613, 2019.

[24] P. Shannon, "Cytoscape: a software environment for integrated models of biomolecular interaction networks," Genome Research, vol. 13, no. 11, pp. 2498-2504, 2003.
[25] M. Tahmasebi Birgani and V. Carloni, “Tumor microenvironment, a paradigm in hepatocellular carcinoma progression and therapy," International Journal of Molecular Sciences, vol. 18, no. 2, p. 405, 2017.

[26] V. Carloni, T. V. Luong, and K. Rombouts, "Hepatic stellate cells and extracellular matrix in hepatocellular carcinoma: more complicated than ever," Liver International, vol. 34, no. 6, pp. 834-843, 2014.

[27] C. C. Schimanski, R. Bahre, I. Gockel et al., "Chemokine receptor CCR7 enhances intrahepatic and lymphatic dissemination of human hepatocellular cancer," Oncology Reports, vol. 16, no. 1, pp. 109-113, 2006.

[28] J.-Y. Shi, L.-X. Yang, Z.-C. Wang et al., "CC chemokine receptor-like 1 functions as a tumour suppressor by impairing CCR7-related chemotaxis in hepatocellular carcinoma," The Journal of Pathology, vol. 235, no. 4, pp. 546-558, 2015.

[29] L. Yang, Y. Chang, and P. Cao, "CCR7 preservation via histone deacetylase inhibition promotes epithelial-mesenchymal transition of hepatocellular carcinoma cells," Experimental Cell Research, vol. 371, no. 1, pp. 231-237, 2018.

[30] A. Rheinländer, B. Schraven, and U. Bommhardt, "CD45 in human physiology and clinical medicine," Immunology Letters, vol. 196, pp. 22-32, 2018.

[31] M. Porcu, M. Kleppe, V. Gianfelici et al., "Mutation of the receptor tyrosine phosphatase PTPRC (CD45) in T-cell acute lymphoblastic leukemia," Blood, vol. 119, no. 19, pp. 4476-4479, 2012.

[32] M. Collette, G. Descamps, C. Pellat-Deceunynck et al., "Crucial role of phosphatase CD45 in determining signaling and proliferation of human myeloma cells," European Cytokine Network, vol. 18, no. 3, pp. 120-126, 2007.

[33] Y.-G. Wei, F. Liu, B. Li et al., "Interleukin-10 gene polymorphisms and hepatocellular carcinoma susceptibility: a meta-analysis," World Journal of Gastroenterology, vol. 17, no. 34, pp. 3941-3947, 2011.

[34] Y. Shi, Q. Song, D. Hu, X. Zhuang, and S. Yu, "Tumorinfiltrating lymphocyte activity is enhanced in tumors with low IL-10 production in HBV-induced hepatocellular carcinoma," Biochemical and Biophysical Research Communications, vol. 461, no. 1, pp. 109-114, 2015.

[35] C. Cui, K. Fu, L. Yang et al., "Hypoxia-inducible gene 2 promotes the immune escape of hepatocellular carcinoma from nature killer cells through the interleukin-10-STAT3 signaling pathway," Journal of Experimental \& Clinical Cancer Research, vol. 38, no. 1, p. 229, 2019.

[36] F. Z. Ouyang, R. Q. Wu, Y. Wei et al., "Dendritic cell-elicited B-cell activation fosters immune privilege via IL-10 signals in hepatocellular carcinoma," Nature Communications, vol. 7, p. 13453, 2016.

[37] K. Fujiwara, T. Higashi, K. Nouso et al., "Decreased expression of B7 costimulatory molecules and major histocompatibility complex class-I in human hepatocellular carcinoma," Journal of Gastroenterology and Hepatology, vol. 19, no. 10, pp. 1121-1127, 2004.

[38] Z.-L. Xiang, Z.-C. Zeng, Z.-Y. Tang et al., "Potential prognostic biomarkers for bone metastasis from hepatocellular carcinoma," The Oncologist, vol. 16, no. 7, pp. 1028-1039, 2011.

[39] L. Chen, M. Li, Q. Li, C.-j. Wang, and S.-q. Xie, "DKK1 promotes hepatocellular carcinoma cell migration and invasion through $\beta$-catenin/MMP7 signaling pathway," Molecular Cancer, vol. 12, no. 1, p. 157, 2013.

[40] Y. Lin, J. Liu, Y. Huang, D. Liu, G. Zhang, and H. Kan, "microRNA-489 plays an anti-metastatic role in human 
hepatocellular carcinoma by targeting matrix metalloproteinase-7," Translational Oncology, vol. 10, no. 2, pp. 211-220, 2017.

[41] K. T. Ng, X. Qi, K. L. Kong et al., "Overexpression of matrix metalloproteinase-12 (MMP-12) correlates with poor prognosis of hepatocellular carcinoma," European Journal of Cancer, vol. 47, no. 15, pp. 2299-2305, 2011.

[42] Z. Z. Liu, L. N. Yan, C. N. Dong et al., "Cytochrome P450 family members are associated with fast-growing hepatocellular carcinoma and patient survival: an integrated analysis of gene expression profiles," Saudi Journal of Gastroenterology, vol. 25 , no. 3, pp. $167-175,2019$.

[43] J. M. Llovet, J. Zucman-Rossi, E. Pikarsky et al., "Hepatocellular carcinoma," Nature Reviews Disease Primers, vol. 2, p. 16018, 2016.

[44] S. Yoo, W. Wang, Q. Wang et al., "A pilot systematic genomic comparison of recurrence risks of hepatitis B virus-associated hepatocellular carcinoma with low- and high-degree liver fibrosis," BMC Medicine, vol. 15, no. 1, p. 214, 2017.

[45] L. Gagnon, M. Leduc, J.-F. Thibodeau et al., "A newly discovered antifibrotic pathway regulated by two fatty acid receptors," The American Journal of Pathology, vol. 188, no. 5, pp. 1132-1148, 2018.

[46] B. Grouix, F. Sarra-Bournet, M. Leduc et al., "PBI-4050 reduces stellate cell activation and liver fibrosis through modulation of intracellular ATP levels and the liver kinase B1/ AMP-activated protein kinase/mammalian target of rapamycin pathway," Journal of Pharmacology and Experimental Therapeutics, vol. 367, no. 1, pp. 71-81, 2018.

[47] C.-H. Chen, L.-Y. Ke, H.-C. Chan et al., "Electronegative low density lipoprotein induces renal apoptosis and fibrosis: STRA6 signaling involved," Journal of Lipid Research, vol. 57, no. 8, pp. 1435-1446, 2016.

[48] H. Lee, H. Jeong, S. Park et al., "Fusion protein of retinolbinding protein and albumin domain III reduces liver fibrosis," EMBO Molecular Medicine, vol. 7, no. 6, pp. 819-830, 2015.

[49] T. A. Wynn and T. R. Ramalingam, "Mechanisms of fibrosis: therapeutic translation for fibrotic disease," Nature Medicine, vol. 18, no. 7, pp. 1028-1040, 2012.

[50] A. E. Thumser, J. B. Moore, and N. J. Plant, "Fatty acid binding proteins," Current Opinion in Clinical Nutrition and Metabolic Care, vol. 17, no. 2, pp. 124-129, 2014.

[51] Y. Shimada, S. Kuninaga, M. Ariyoshi et al., "E2F8 promotes hepatic steatosis through FABP3 expression in diet-induced obesity in zebrafish," Nutrition \& Metabolism, vol. 12, p. 17, 2015.

[52] V. Stiegelbauer, P. Vychytilova-Faltejskova, M. Karbiener et al., "miR-196b-5p regulates colorectal cancer cell migration and metastases through interaction with HOXB7 and GALNT5," Clinical Cancer Research, vol. 23, no. 17, pp. 5255-5266, 2017.

[53] H. Guo, L. Zhao, B. Shi et al., "GALNT5 uaRNA promotes gastric cancer progression through its interaction with HSP90," Oncogene, vol. 37, no. 33, pp. 4505-4517, 2018.

[54] B. Liang, M. Shahbaz, Y. Wang et al., "Integrin 6-targeted immunoliposomes mediate tumor-specific drug delivery and enhance therapeutic efficacy in colon carcinoma," Clinical Cancer Research, vol. 21, no. 5, pp. 1183-1195, 2015.

[55] R. A. de Almeida, T. Heuser, R. Blaschke, C. R. Bartram, and J. W. G. Janssen, "Control of MYEOV protein synthesis by upstream open reading frames," Journal of Biological Chemistry, vol. 281, no. 2, pp. 695-704, 2006.
[56] L. Fang, S. Wu, X. Zhu et al., "MYEOV functions as an amplified competing endogenous RNA in promoting metastasis by activating TGF- $\beta$ pathway in NSCLC," Oncogene, vol. 38, no. 6, pp. 896-912, 2019.

[57] G. Lawlor, P. P. Doran, P. MacMathuna, and D. W. Murray, "MYEOV (myeloma overexpressed gene) drives colon cancer cell migration and is regulated by PGE2," Journal of Experimental \& Clinical Cancer Research, vol. 29, no. 1, p. 81, 2010.

[58] N. Weinhold, A. Jacobsen, N. Schultz, C. Sander, and W. Lee, "Genome-wide analysis of noncoding regulatory mutations in cancer," Nature Genetics, vol. 46, no. 11, pp. 1160-1165, 2014.

[59] J. Zucman-Rossi, A. Villanueva, J.-C. Nault, and J. M. Llovet, "Genetic landscape and biomarkers of hepatocellular carcinoma," Gastroenterology, vol. 149, no. 5, pp. 1226-1239, 2015. 


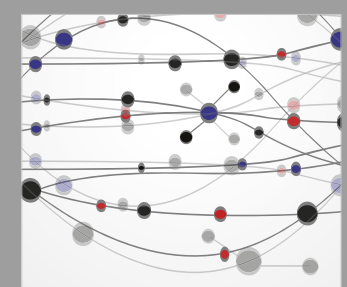

The Scientific World Journal
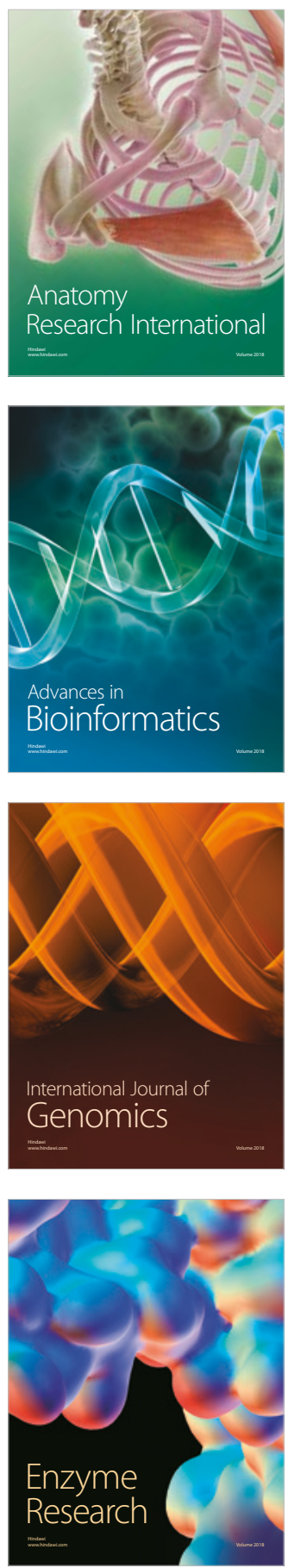
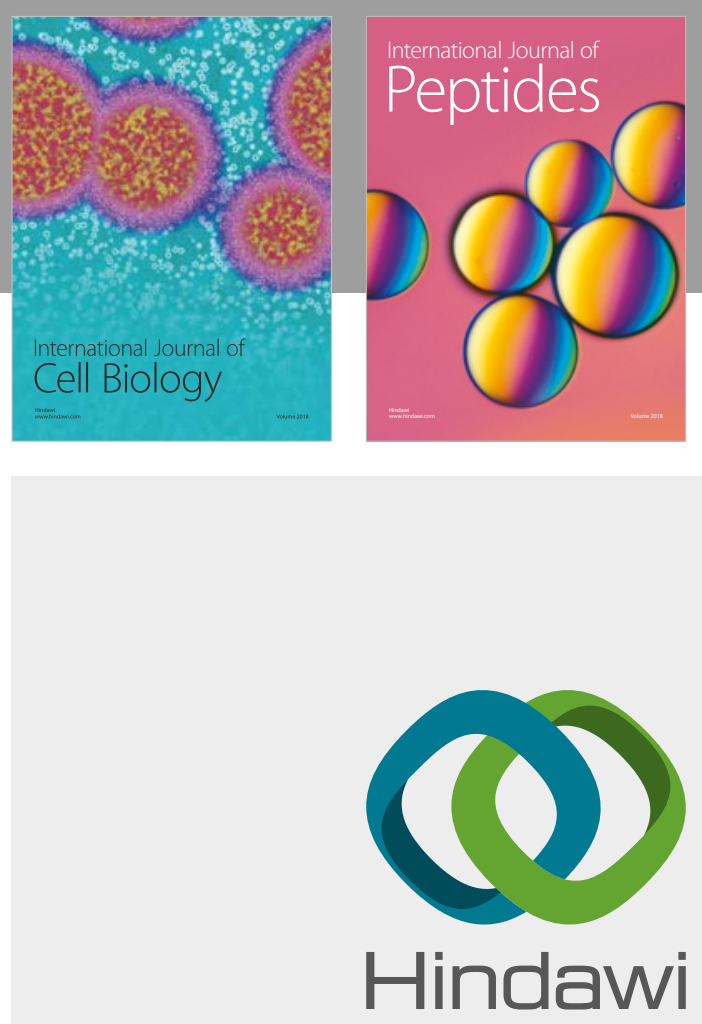

Submit your manuscripts at

www.hindawi.com
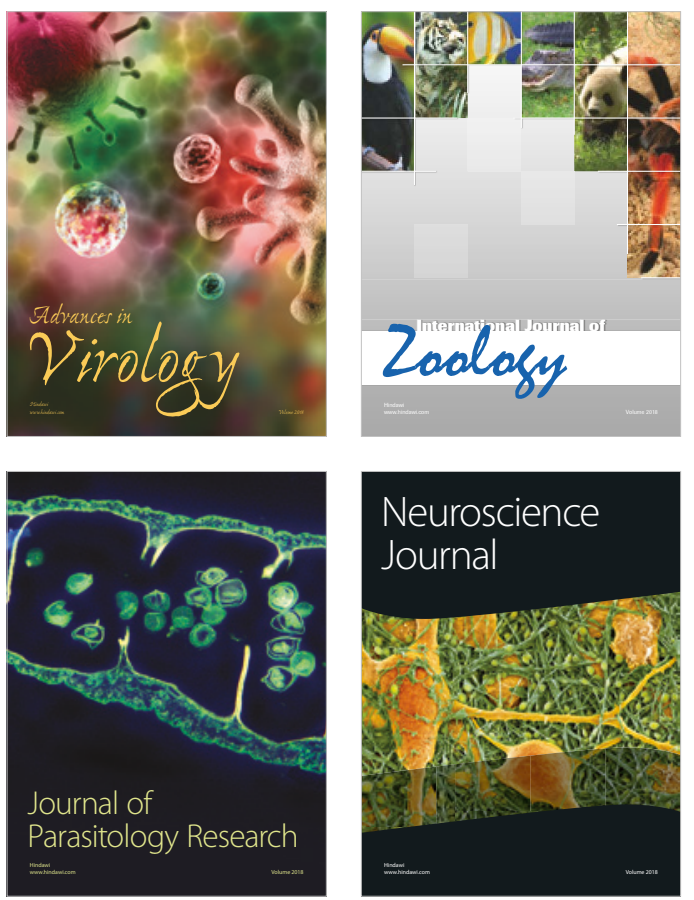
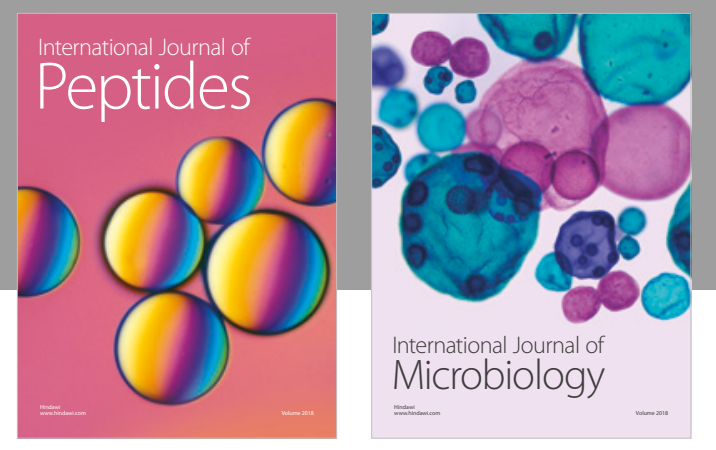

nternational Journal of Microbiology
Journal of
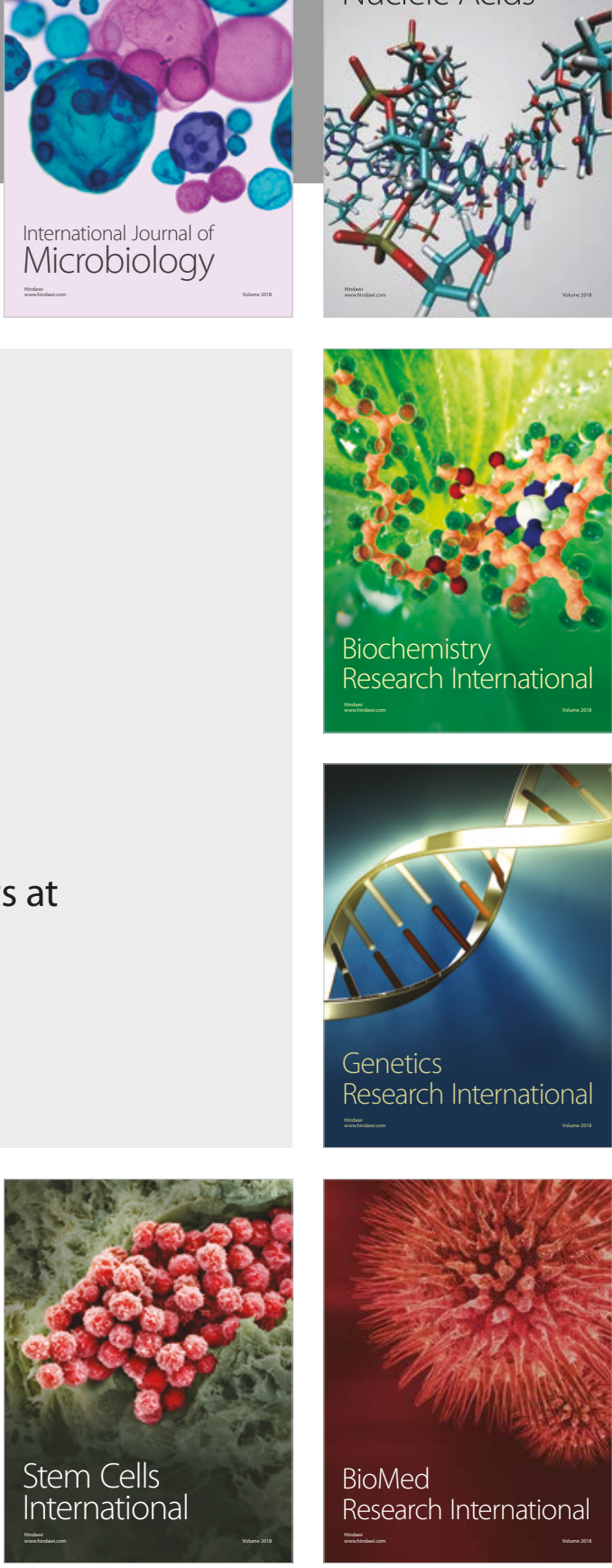
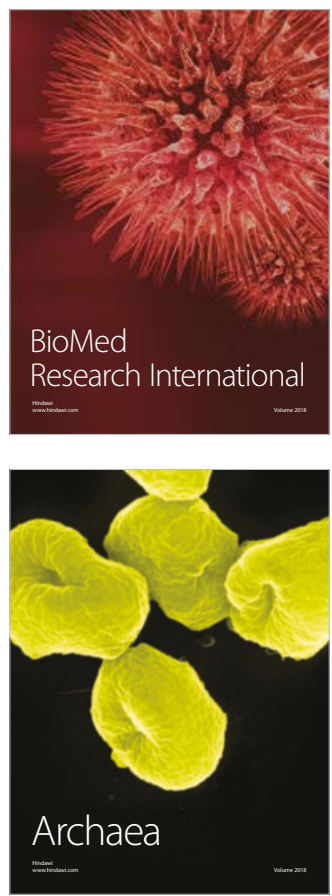